\title{
83 MINDING THE GAP - DROP-OUT OF HEPATITIS C PCR POSITIVE PATIENTS BETWEEN DIAGNOSIS AND ATTENDANCE AT THE LIVER CLINIC
}

A McCurley ${ }^{1}$, S Murray ${ }^{1}$, M McCartney ${ }^{1}$, P Coyle ${ }^{2}$, N McDougall ${ }^{3}$, I Cadden ${ }^{3}$ The Liver Unit, 1st Floor East Wing, Royal Victoria Hospital, Grosvenor Road, Belfast, Co Antrim, Northern Ireland

10.1136/gutjnl-2013-305143.83

Introduction In 2009 we identified a sizeable gap between those diagnosed with Hepatitis $\mathrm{C}(\mathrm{HCV})$ and those seen at clinic. The Northern Ireland Managed Clinical Network (MCN) has reviewed all polymerase chain reaction (PCR) positive cases with respect to referral to/attendance at clinic.

Aims/Background To review referral to/attendance at Liver Clinic for confirmed HCV PCR positive cases.

Method Confirmed cases between September 2009 and June 2012 were cross-referenced with the Patient Administration System to assess clinic attendance. In cases why patients were not referred, the originator of the test was contacted, for an explanation and to encourage referral to the clinic.

Results 219 cases were confirmed. 118 (53\%) cases were referred before MCN intervention. After this, 193 referrals were received (88\%). 26 (12\%) individuals were never referred. 6 persons died following referral, leaving 187 referred cases. 149(77\%) individuals currently attend the liver clinic; 1 awaits appointment. 42\% of referrals were from ethnic minority groups. Annual nonattendance rates dropped from $22 \%$ in 2009 to $16 \%$ in 2011.37 patients referred did not attend clinic; 18 from ethnic minority backgrounds. 19 were from UK/Northern Ireland.

There was no significant difference between non-attendance rate amongst ethnic minority groups $(22.8 \%)$ and for those with a UK/Northern Ireland origin (17.9\%) (Fishers Exact Test; $\mathrm{p}=$ 0.46).

Conclusion Involvement of the NI Hepatitis B\&C MCN has increased rates of referral of HCV PCR positive patients for assessment. Surprisingly, ethnic background did not impact upon the rate of clinic non-attendance. 